\section{Global prevalence of childhood cataract: a systematic review}

S Sheeladevi, JG Lawrenson, AR Fielder and CM Suttle

\begin{abstract}
Childhood cataract is an avoidable cause of visual disability worldwide and is a priority for VISION 2020: The Right to Sight. There is a paucity of information about the burden of cataract in children and the aim of this review is to assess the global prevalence of childhood cataract. The methodology for the review followed the Preferred Reporting Items for Systematic Reviews and Meta-Analyses (PRISMA) guidelines. We performed a literature search for studies reporting estimates of prevalence or incidence of cataract among children (aged $<\mathbf{1 8}$ years) at any global location using the Cochrane Library, Medline and Embase up to January 2015. No restrictions were imposed based on language or year of publication. Study quality was assessed using a critical appraisal tool designed for systematic reviews of prevalence. Twenty prevalence and four incidence studies of childhood cataract from five different geographical regions were included. The overall prevalence of childhood cataract and congenital cataract was in the range from 0.32 to $22.9 / 10000$ children (median $=1.03$ ) and 0.63 to $9.74 / 10000$ (median $=1.71$ ), respectively. The incidence ranged from 1.8 to $3.6 / 10000$ per year. The prevalence of childhood cataract in low-income economies was found to be 0.42 to 2.05 compared with 0.63 to $13.6 / 10000$ in high-income economies. There was no difference in the prevalence based on laterality or gender. This review highlights substantial gaps in the epidemiological knowledge of childhood cataract worldwide, particularly from low and lower middle-income economies. More studies are needed using standard definitions and case ascertainment methods with large enough sample sizes. Eye (2016) 30, 1160-1169; doi:10.1038/eye.2016.156; published online 12 August 2016
\end{abstract}

Centre for Public Health Research, Division of Optometry and Visual Science, School of Health Sciences, City University London, London, UK

Correspondence:

S Sheeladevi, Centre for Public Health Research, Division of Optometry and Vision Science, School of Health Sciences, City University London, London EC1V OHB, UK

Tel: +44 2070408874 ;

Fax: +44 2070404310

E-mail: Sheeladevi.sethu@ city.ac.uk

Received: 30 March 2016 Accepted in revised form: 24 June 2016

Published online:

12 August 2016

\section{Introduction}

Cataract is defined as any opacity of the crystalline lens of the eye, which impedes the image clarity causing reduced visual acuity and impaired contrast sensitivity. Cataract in children may be congenital or acquired, unilateral or bilateral ${ }^{1}$ and in the majority of cases is treatable. Although it is rare, childhood cataract is one of the most important causes of blindness and severe visual impairment in children and is responsible for $5-20 \%$ of pediatric blindness worldwide. ${ }^{2}$ It is estimated that 200000 children worldwide are blind due to cataract, and that 20000-40 000 children are born each year with congenital cataract. ${ }^{3}$ Cataract blindness in children presents an enormous problem to developing countries in terms of human morbidity, economic loss, and social burden. ${ }^{4}$

Studies conducted in schools for the blind have investigated the various causes of childhood blindness. Previous reports from West Africa, South India, and Chile showed that lens abnormalities accounted for $15.5 \%, 7.4 \%$, and $9.2 \%$ of blindness in such schools. ${ }^{5}$ Similar studies conducted in Malawi, Kenya and Uganda found that blindness was caused by unoperated cataract in $13.1 \%, 9.1 \%$, and $27.6 \%$ of children, respectively. ${ }^{6}$ In Ethiopia, unoperated cataract or aphakia accounted for $9.2 \%$ of blindness in schools for the blind. ${ }^{7}$ With significant reductions in some of the preventable causes of blindness such as measles and vitamin A deficiency, cataract has become the major cause of treatable blindness in children in developing countries. ${ }^{8}$

Reliable region-specific data on the prevalence and incidence of childhood cataract is important as a basis for policy decisions, including the evidence-based allocation of resources. Cost and logistics limit the feasibility of the large-scale data gathering required for prevalence estimates. The key informant method, in which key community members are trained to identify people within the community with a given health condition, was introduced to calculate prevalence based on a ratio of cases identified and an estimate of the total number at risk in a 
particular geographical area. However, few studies have used this method to date, ${ }^{9,10}$ and there is a paucity of epidemiological information about cataract in children globally. Thus, there is a lack of evidence to guide policy related to childhood cataract. Currently, there are no systematic reviews on the question of prevalence and incidence of childhood cataract. The aim of this study is to systematically review existing research to determine a reliable estimate of global prevalence and incidence of congenital (from birth) and acquired (due to trauma or disease) cataract in children.

\section{Methods}

\section{Search strategy and selection criteria}

We followed the Preferred Reporting of Items for Systematic Reviews and Meta-Analyses (PRISMA) guidelines. The Cochrane Library, Medline, and Embase were searched (the date of last search was January 2015 via OVID and EBSCOHOST) using the following search terms formatted for OVID search: ('Child”' [All Fields] OR 'infan*' [Title] OR 'p?ediatric ${ }^{* \prime}$ [Title] OR ' adolescen*' [Title] OR 'teenage*' [Title] OR 'juvenile ${ }^{* \prime}$ [Title] OR 'minor' [Title] OR 'young people') AND ('Cataract' [Abstract] OR 'lens*' [Abstract] OR 'near opacity*' [Abstract]) AND ('prevalence' [Abstract] OR 'incidence' [Abstract] OR 'epidemiology' [Abstract]. No restrictions were imposed based on language or year of publication. Bibliographies of related articles were checked to identify additional potentially relevant reports. The World Health Organisation website was searched for program reports and government documentation. The protocol for this review has been registered and published on the Prospero database (http:/ /www.crd.york.ac.uk/PROSPERO/ prospero.asp; reference number CRD42014014909).

\section{Inclusion and exclusion criteria}

We included all studies at any global location which estimated the prevalence and/or incidence of cataract among children (aged less than 18 years). In this context, prevalence indicates the number of children in a population that have cataract at a given point of time divided by those at risk (the total number of children in the population sample). Incidence indicates how many new cases of cataract occur in children under 18 years within a defined period of time. For estimating prevalence, data from non-random samples (eg, from schools for the blind) or based on self-report were excluded. For incidence studies, no exclusion criteria were imposed.

\section{Quality assessment and data extraction}

One reviewer (SS) conducted the search and all of the studies derived from the search were independently assessed by two reviewers (SS and CMS) for inclusion initially based on title and abstract content followed by full-text review of potentially eligible studies, using the criteria outlined above. Any discrepancies were discussed and resolved by consensus. After this process the included studies were assessed for methodological quality based on the full-published paper independently by both SS and CMS using the prevalence critical appraisal instrument developed by Munn et al. ${ }^{11}$ Criteria used to judge quality are provided in Figure 1. Data were independently extracted from eligible studies by two reviewers (SS and CMS), and the resulting data were verified by a third reviewer (JGL). All the quantitative data synthesis was carried out using Open Meta Analyst. $^{12}$

\section{Statistical analysis}

We intended to calculate a pooled estimate of the global prevalence of childhood cataract (congenital and acquired) and the prevalence of congenital cataract only. In addition, we obtained an estimate of the prevalence of childhood cataract according to the country's economic status across included studies. Heterogeneity was assessed using the Cochran's Q chi-squared statistic and by calculating the $I^{2} .{ }^{13,14}$ Prevalence was assessed for geographical location according to income status, defined according to the gross national income per capita per year and calculated using the World Bank Atlas method (http: / / data.worldbank.org/about/countryand-lending-groups). Correlation tests were used to correlate variables with $P<0.05$ considered as statistically significant.

\section{Results}

Out of a total of 677 potentially relevant titles/abstracts, 44 full-text articles were identified from searches of bibliographic databases, with 24 of these meeting the inclusion criteria. The PRISMA flow diagram is shown in Figure 2. The majority of the studies reviewed were in English $(n=41)$, two in Mandarin and one in Portuguese. Reasons for exclusion of the other 20 studies are reported in Appendix 1 (available as Supplementary Information).

Half of the included studies $(n=13)$ were published between 2004 and 2014 and all of the included studies were published between 1988 and 2014. Twenty studies reported data on prevalence ${ }^{15-34}$ and four studies reported incidence. ${ }^{35-38}$ 


\begin{tabular}{|c|c|c|c|c|c|c|c|c|c|c|}
\hline Study & 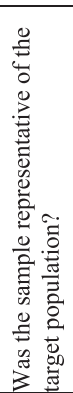 & 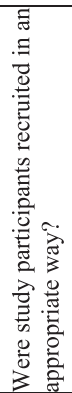 & 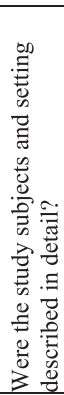 & 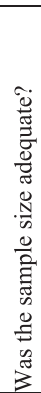 & 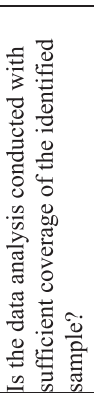 & 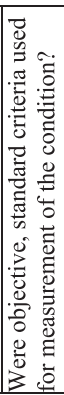 & 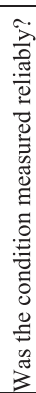 & 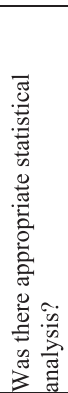 & 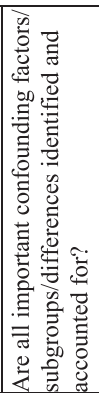 & 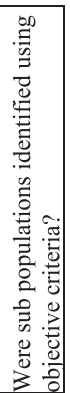 \\
\hline
\end{tabular}

\section{Prevalence}

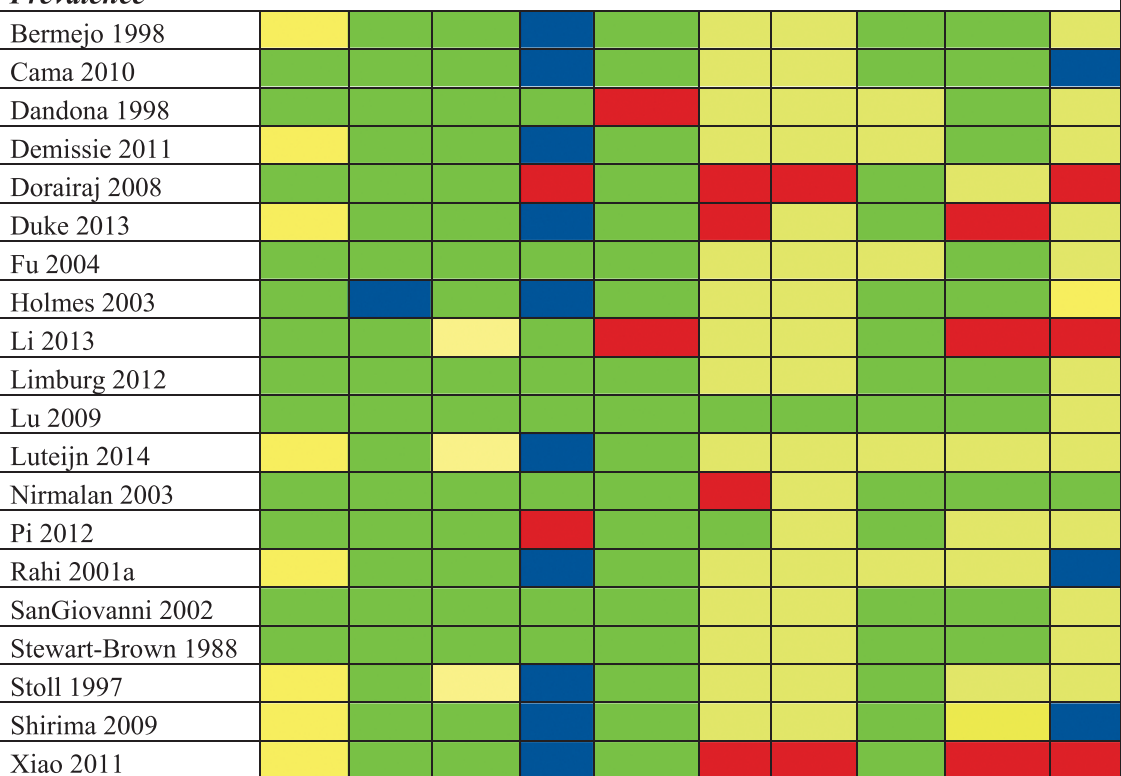

Xiao 2011

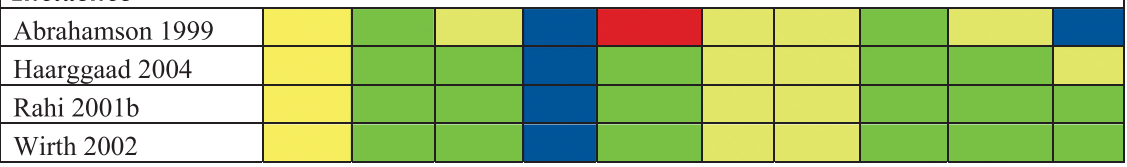

$$
\begin{aligned}
& \square \text { - Yes } \\
& \text { - No } \\
& \square \text { - Unclear } \\
& \text { - Not applicable }
\end{aligned}
$$

Figure 1 Quality assessment of the 24 included studies.

The included studies represented five geographical regions including Europe and Central Asia $(n=8)$, South Asia $(n=3)$, East Asia and Pacific $(n=8)$, Sub-Saharan Africa $(n=3)$, and North America $(n=2)$.

Sample sizes in the included studies varied greatly, ranging from small samples in regional cross-sectional studies to analyses of large data sets derived from national registries. The methods used for case definition also varied between studies: from lens opacities detected following an ocular examination to cataract causing varying degrees of unilateral or bilateral visual impairment. The characteristics of included studies are presented in Table 1 and the results of the quality assessment summarised in Figure 1. Studies were generally of moderate to good methodological quality, although they were often poorly reported.

A considerable degree of heterogeneity was found between the 20 studies reporting prevalence of childhood cataract (Cochran's $Q$-test, $P<0.01 ; I^{2}=94 \%$; see Figure 3). Given the heterogeneity in prevalence estimates and differences in study design and methods of case ascertainment, we did not perform a meta-analysis. The 


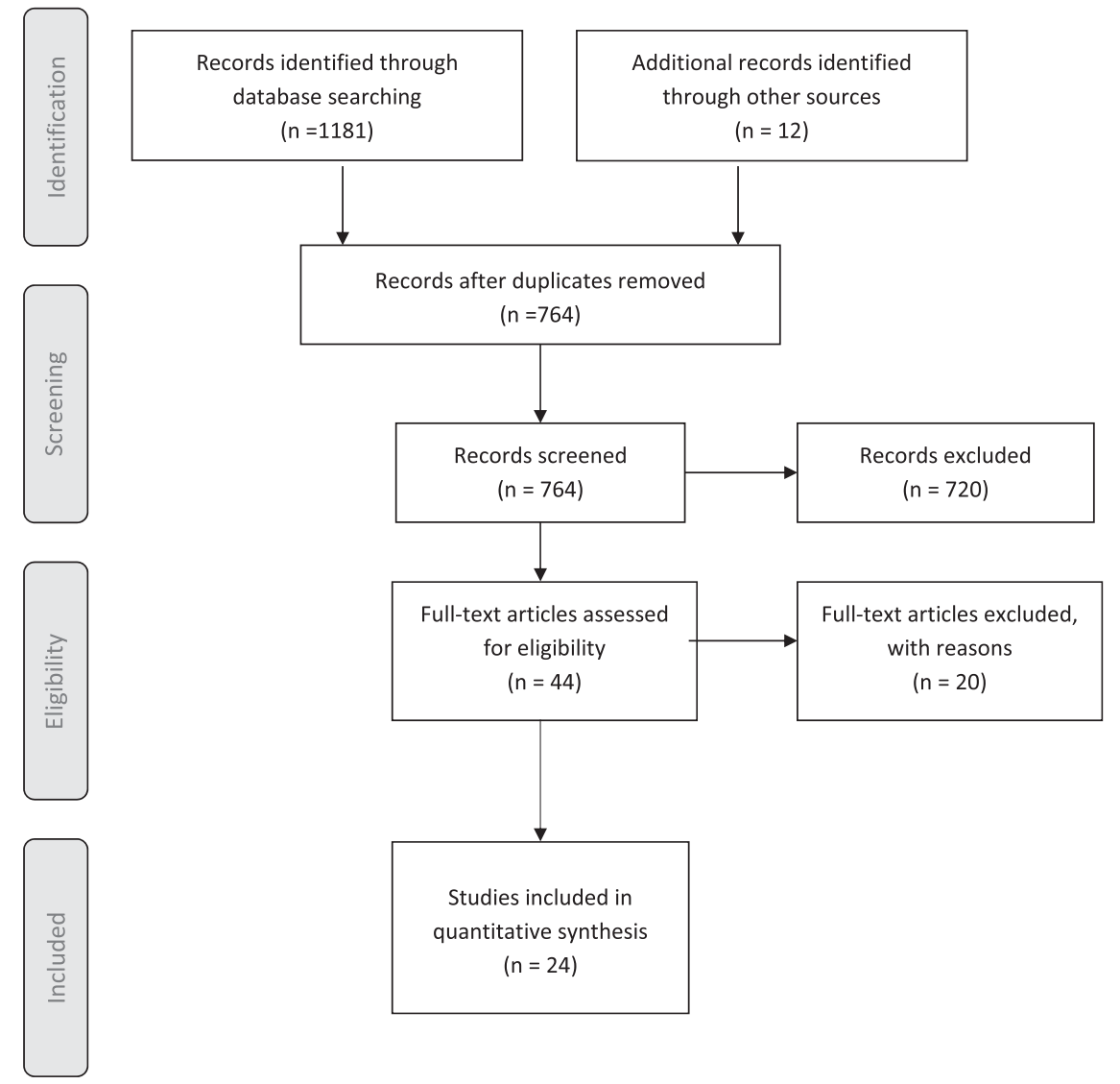

Figure 2 Summary of review strategy_PRISMA Flow Diagram.

overall prevalence of childhood cataract ranged from 0.32 to 22.9 per 10000 (median 1.03/10 000) and 0.63 to 9.74 per 10000 (median 1.71/10 000) for congenital cataract based on 13 studies that reported congenital cataract.

The prevalence in low-income and lower middleincome economies ranged from 0.42 to 2.05 per 10000 and 0.32 to 8.49 per 10000 , respectively; in upper middleincome economies, it was from 0.74 to 22.7 per 10000 ; and in high-income economies, it was from 0.63 to 13.6 per 10000 .

Prevalence by laterality (unilateral or bilateral) was reported in four studies $22,30,32,34$ and three studies reported data on traumatic cataract. ${ }^{25,28,30}$ Overall, the reported prevalence of unilateral and bilateral cataract was similar $(P=0.21)$. Prevalence was reported according to gender in five studies $22,24,30,31,34$ and there was no difference in prevalence of childhood cataract by gender $(P=0.48)$.

Incidence was reported in four studies ${ }^{35-38}$ and ranged from 1.8 to 3.6 per 10000 per annum.

Laterality was reported in two of these studies ${ }^{35,37}$ and gender breakdown was reported in three studies. ${ }^{35-37}$ The incidence of cataract by laterality $(P=0.35)$ and gender $(P=0.76)$ was similar.

\section{Discussion}

To our knowledge, this is the first systematic review of prevalence and incidence studies of childhood cataract. The review included 20 prevalence studies and four incidence studies from five different geographical regions that were published between 1988 and 2014. The median prevalence of childhood cataract was 1.03 per 10000 (range $0.32-22.9 / 10000$ ) children. Over $90 \%$ of cataracts were classified as congenital or developmental.

It is not clear whether the wide range in reported prevalence values reflects true variances between populations or whether this is due to differences in methodology and/or case definitions used in the included studies. For example, birth cohort studies would have missed developmental cataracts; studies using visual acuity of the better-seeing eye to identify those requiring further evaluation would have missed unilateral cataract and those who have successfully undergone cataract surgery. Moreover, studies classifying cataract as any lens opacity would have a higher prevalence than those using a definition of visual impairment or blindness because of cataract. Reliability of diagnosis is of fundamental 


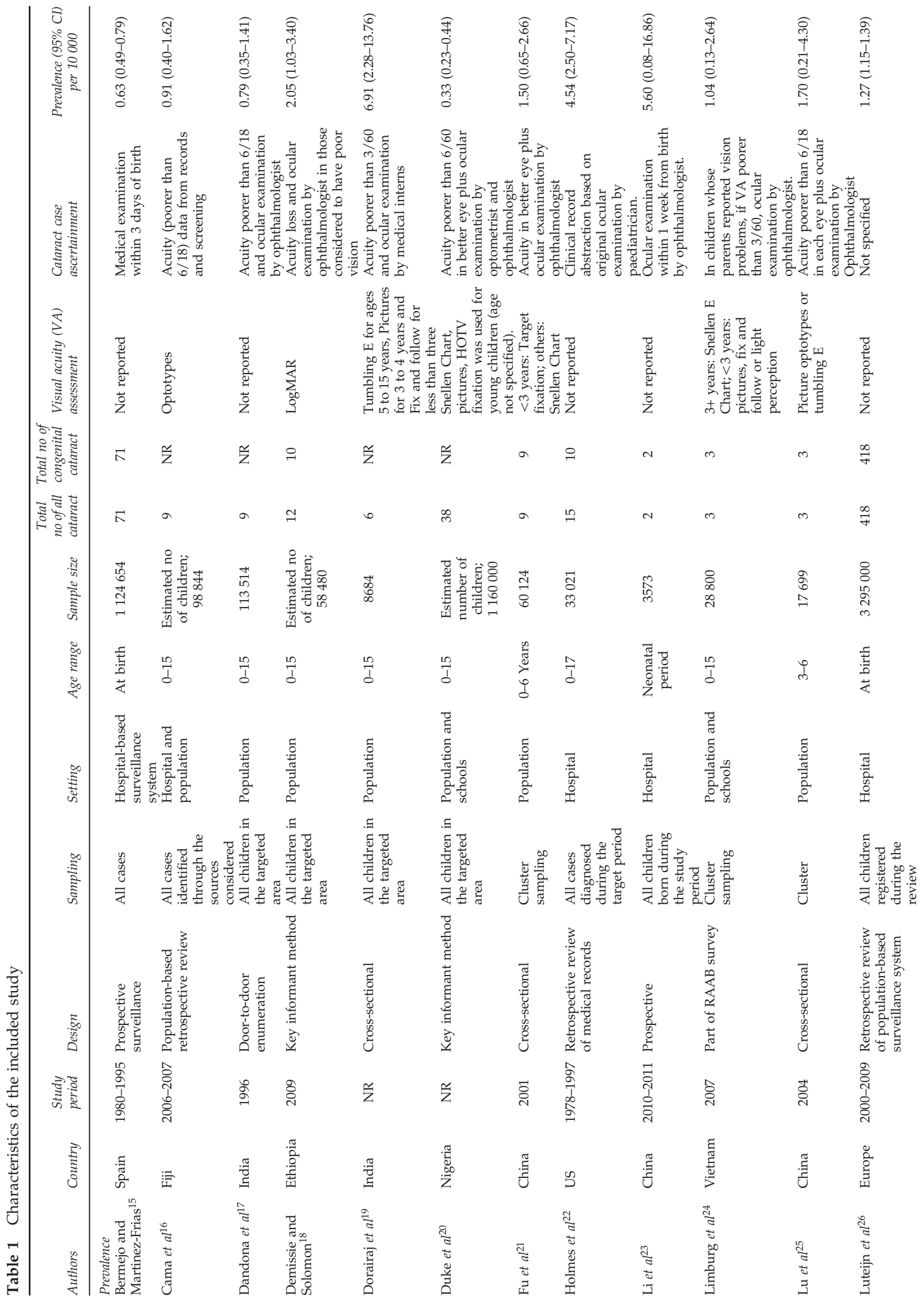




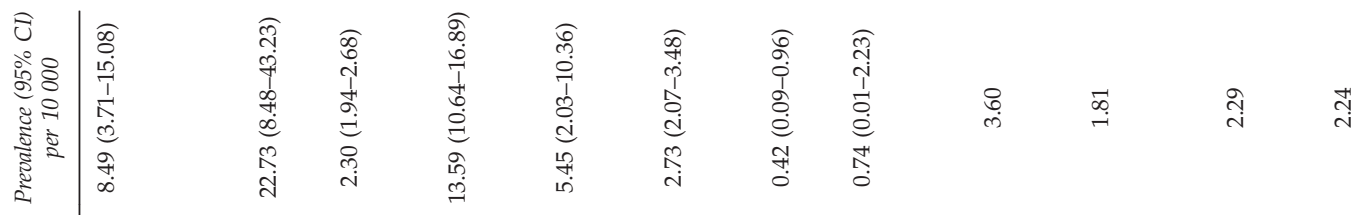

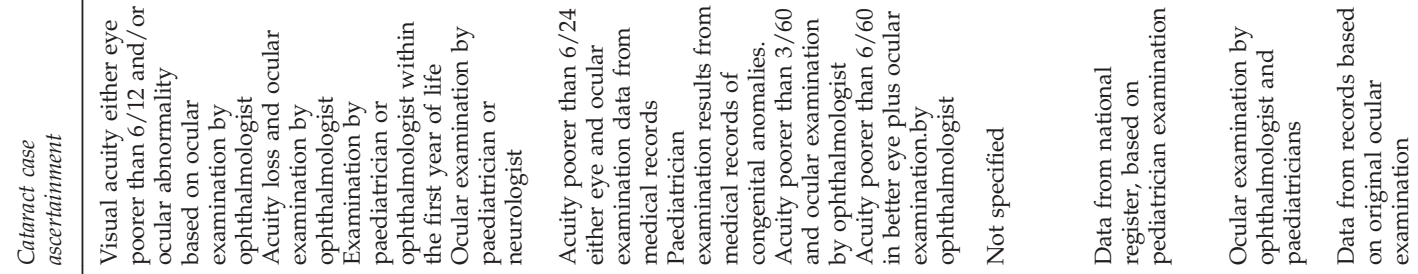

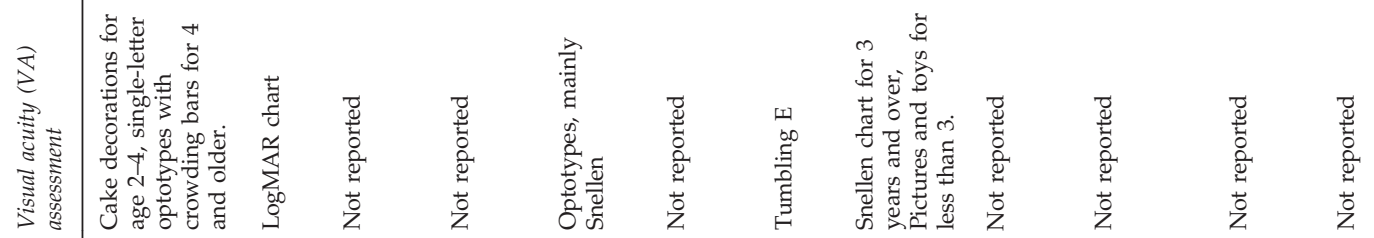

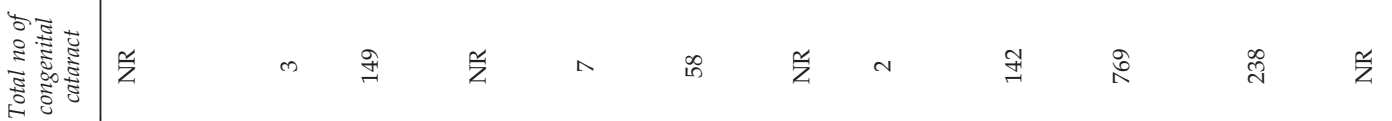

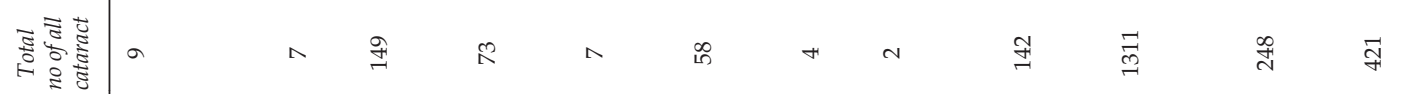

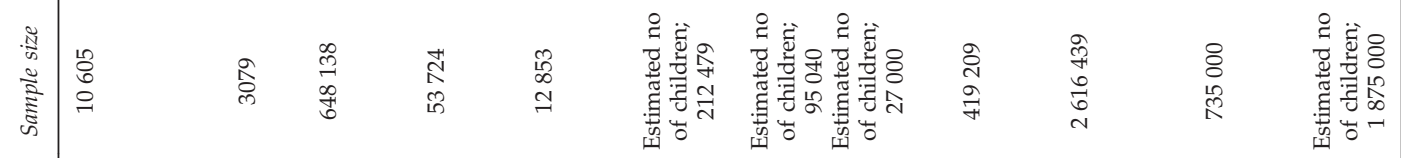

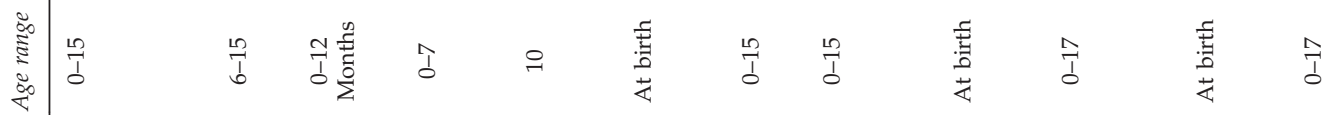

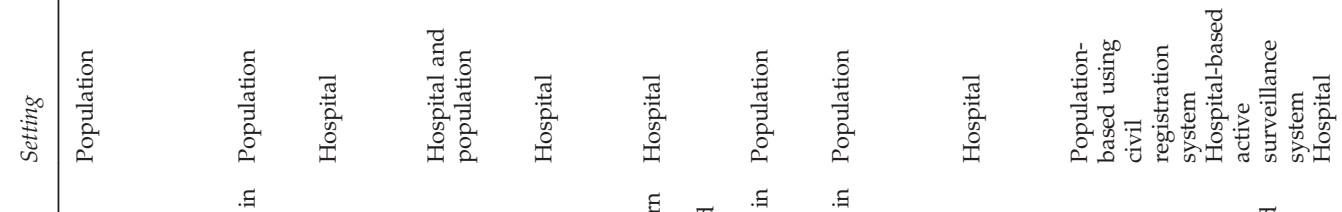

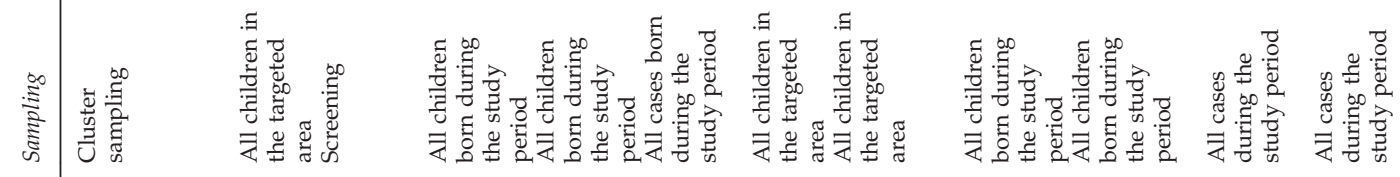

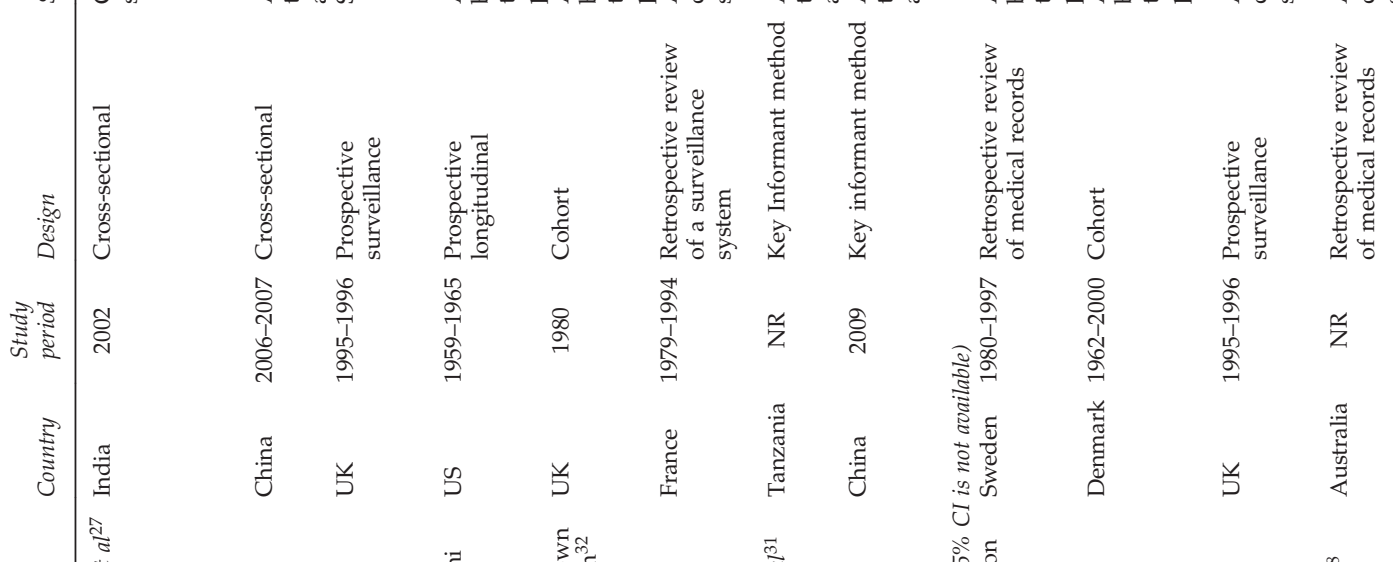
舀

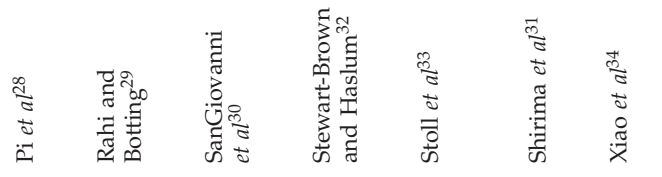
Un 


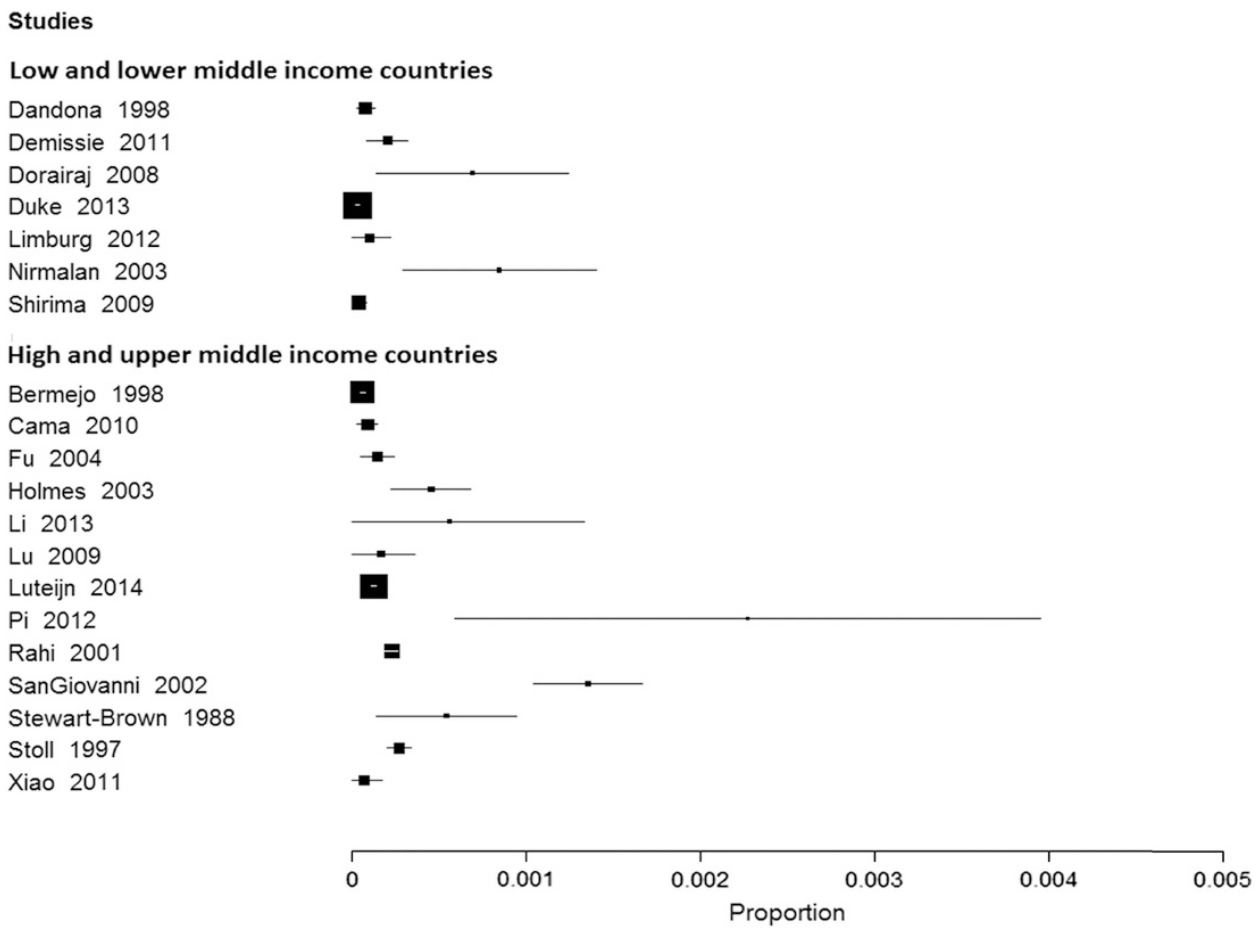

Figure 3 Forest Plot on prevalence of Childhood cataract in low- and lower middle-income countries compared with high- and higher middle-income countries (proportions with 95\% confidence interval). For each study, the size of the symbol corresponds to the sample size.

importance in a prevalence study. In the studies we have reviewed, a detailed description of the diagnostic method was often lacking. For example, some studies indicated that slit lamp biomicroscopy was used, but did not explain on what basis (eg, grading scheme) cataract was diagnosed. It has been suggested that both subjective and objective evaluations of infantile cataracts are important to predict its effects on visual performance. ${ }^{39}$

Various methods have been developed and validated for the assessment of vision in infants and young children. ${ }^{40}$ In most of the studies included here it was unclear whether the methods used would provide a reliable assessment of vision, and in general basic methods such as infants' detection of small objects, or perception of light were used. These methods cannot provide an accurate indication of acuity, and simple, affordable methods such as preferential looking cards would provide a better means of gauging the severity of vision loss in prevalence studies on childhood cataract.

It has been previously reported that the prevalence of blindness due to childhood cataract is 10 times higher in low-income economies compared with high-income economies. ${ }^{3}$ This is primarily due to inadequate healthcare systems, malnutrition and higher rates of perinatal infections, for example, rubella. The present findings do not agree with this, and suggest higher prevalence estimates in high-income than lower income economies. This may reflect the fact that the majority of included studies in high-income countries did not use visual acuity as part of the case definition of cataract. Studies using visual acuity to define cases were mostly focused on children with blindness or visual impairment, and would identify cases with severe vision loss, missing those with unilateral or moderate vision loss. Such studies may therefore underestimate cataract prevalence. In addition, the relatively low estimate in low-income economies may be due in part to the association between conditions causing blindness and high under 5 mortality rates in these regions. For example, the survival rate of children with blinding conditions such as vitamin A deficiency is lower in countries with high under-five mortality rates. ${ }^{41}$ As outlined above, our prevalence estimates do not show higher prevalence in low income economies and these findings suggest that more studies with adequate, representative samples are needed with a common case definition to more accurately estimate the prevalence of childhood cataract. This is particularly challenging in lowincome countries due to costs and the logistics involved, compared with high-income economies where national registries and surveillance systems facilitate epidemiological data collection. ${ }^{26,29,36}$

It is worth noting that heterogeneity of reported prevalence varies considerably within as well as between 
regions. If we take China (an upper middle-income economy) as one example, prevalence studies included in this review were carried out in Beijing (prevalence $1.7 / 10000),{ }^{25}$ South-Eastern China $(0.7 / 10000),{ }^{34}$ South-Western China (5.6/10 000), ${ }^{23}$ across all states $(1.5 / 10000)^{21}$ and in Western China $(22.7 / 10000){ }^{28}$ The authors of the latter study commented that Western China is relatively undeveloped compared with other regions in the country, and this may illustrate the existence of a range of health-care provision and prevalence within one country.

Incidence studies included in this review were conducted in Sweden, ${ }^{35}$ Denmark, ${ }^{36}$ the UK, ${ }^{37}$ and Australia. ${ }^{38}$ These are all high-income economies; we found no incidence studies based in low- to middleincome economies.

Another important finding from this review is that both bilateral and unilateral cataract have similar prevalence, so about half of the cases are bilateral and about half are unilateral. Both have significant impact on vision in different ways. Unoperated bilateral cataract has the obvious impact of reducing vision in both eyes, thus causing severe visual impairment and blindness.

Unilateral cataract, on the other hand, has seemingly less impact, since it affects vision in only one eye, leaving the fellow eye able to provide unimpeded vision. However, it is important to note that bilateral visual deprivation during early childhood has a less severe impact on visual system development than unilateral deprivation. ${ }^{42}$ In particular, amblyopia is a condition in which vision is abnormal (eg, reduced acuity in one eye and poor binocular depth perception) as a result of abnormal visual input during early life. Treatment to correct visual abnormality is more successful in early childhood, ${ }^{43}$ during a period of visual system plasticity, than later, so early diagnosis and management is important for any childhood condition in which vision is impeded. ${ }^{44,45}$ Thus, early treatment in both cases is important, to remove the cataract as an impediment to vision and provide refractive correction. Consistent with this, the appropriate provision of surgery for congenital cataracts is one of the specific disease control objectives in the Vision 2020 program to control blindness in children. ${ }^{2,46}$

To conclude, this review highlights substantial gaps in the epidemiological knowledge of childhood cataract worldwide, particularly from low- and lower middle-income economies, where the burden of childhood cataract is presumed to be high. Using the median prevalence of 1.03/10 000 children and an estimated $26 \%$ of the global population aged $<15$ years (http:/ / www.worldometers.info/world-population/ world-population-gender-age.php) (1.86 billion children in this age group), this would translate to approximately
191000 cases of childhood cataract worldwide. Similarly, using the median incidence of 1.69 per 10000 , which translates to around 314000 new childhood cataract (both congenital and developmental) cases every year. Future studies should report age, gender and ethnicity-specific estimates of incidence and prevalence, and attempt to standardise epidemiological methods and case definitions (particularly incorporating visual impairment). These estimates could then inform policy decisions to prioritise funding of programs to reduce visual impairment and blindness due to childhood cataract at regional and global levels. Delivering timely surgical intervention ${ }^{6}$ and appropriate follow-up after surgery would avoid blindness in children due to cataract, as emphasised and advocated by the Vision 2020 initiative: The Right to Sight Initiative. ${ }^{47}$

\section{Conflict of interest}

The authors declare no conflict of interest.

\section{Author contributions}

SS contributed to protocol design, study design, the literature review, quality assessment, the data extraction, statistical analysis, the data interpretation, article preparation and correspondence. CMS contributed to protocol design, study design, the literature review, quality assessment, the data extraction, statistical analysis, the data interpretation and article review. JGL contributed to protocol design, the data analysis, the data interpretation and article review. ARF contributed to study design and article review.

\section{Acknowledgements}

We thank Dr Angela Lai and Bruno Fidalgo for help with translation.

\section{References}

1 Gralek M, Kanigowska K, Seroczynska M. Cataract in children-not only an ophthalmological problem. Med Wieku Rozwoj 2007; 11(2 Pt 2): 227-230.

2 Gilbert C, Foster A. Childhood blindness in the context of VISION 2020-the right to sight. Bull World Health Organ 2001; 79(3): 227-232.

3 Foster A, Gilbert C, Rahi J. Epidemiology of cataract in childhood: a global perspective. J Cataract Refract Surg 1997; 23(Suppl 1): 601-604.

4 Wilson ME, Pandey SK, Thakur J. Paediatric cataract blindness in the developing world: surgical techniques and intraocular lenses in the new millennium. Br J Ophthalmol 2003; 87(1): 14-19.

5 Gilbert CE, Canovas R, Kocksch de Canovas R, Foster A. Causes of blindness and severe visual impairment in 
children in Chile. Dev Med Child Neurol 1994; 36(4): 326-333.

6 Gilbert C, Awan H. Blindness in children. BMJ 2003; 327(7418): 760-761.

7 Kello AB, Gilbert C. Causes of severe visual impairment and blindness in children in schools for the blind in Ethiopia. Br J Ophthalmol 2003; 87(5): 526-530.

8 Waddell KM. Childhood blindness and low vision in Uganda. Eye 1998; 12(Pt 2): 184-192.

9 Muhit MA. Finding children who are blind. Community Eye Health 2007; 20(62): 30-31.

10 Muhit MA, Shah SP, Gilbert CE, Hartley SD, Foster A. The key informant method: a novel means of ascertaining blind children in Bangladesh. Br J Ophthalmol 2007; 91(8): 995-999.

11 Munn Z, Moola S, Riitano D, Lisy K. The development of a critical appraisal tool for use in systematic reviews addressing questions of prevalence. Int J Health Policy Manag 2014; 3(3): 123-128.

12 Wallace BC, Dahabreh IJ, Trikalinos TA, Lau J, Trow P, Christopher HS. Closing the gap between methodologists and end-users: $\mathrm{R}$ as a computational back-end. J Stat Softw 2012; 49: 5.

13 Higgins JP, Thompson SG. Quantifying heterogeneity in a meta-analysis. Stat Med 2002; 21(11): 1539-1558.

14 Higgins JP, Thompson SG, Deeks JJ, Altman DG. Measuring inconsistency in meta-analyses. BMJ 2003; 327(7414): 557-560.

15 Bermejo E, Martinez-Frias ML. Congenital eye malformations: clinical-epidemiological analysis of 1,124,654 consecutive births in Spain. Am J Med Genet 1998; 75(5): 497-504.

16 Cama AT, Sikivou BT, Keeffe JE. Childhood visual impairment in Fiji. Arch Ophthalmol 2010; 128(5): 608-612.

17 Dandona L, Williams JD, Williams BC, Rao GN. Populationbased assessment of childhood blindness in Southern India. Arch Ophthalmol 1998; 116(4): 545-546.

18 Demissie BS, Solomon AW. Magnitude and causes of childhood blindness and severe visual impairment in Sekoru District, Southwest Ethiopia: a survey using the key informant method. Trans R Soc Trop Med Hyg 2011; 105(9): 507-511.

19 Dorairaj S, Bandrakalli P, Shetty CRV, Misquith D, Ritch R. Childhood blindness in a rural population of Southern India: prevalence and etiology. Ophthalmic Epidemiol 2008; 15(3): 176-182.

20 Duke R, Otong E, Iso M, Okorie U, Ekwe A, Courtright P et al. Using key informants to estimate prevalence of severe visual impairment and blindness in children in Cross River State, Nigeria. J AAPOS 2013; 17(4): 381-384.

21 Fu P, Yang L, Bo S-Y, Na X. A national survey on low vision and blindness of $0-6$ years old children in China. Zhonghua Yi Xue Za Zhi 2004; 84(18): 1545-1548.

22 Holmes JM, Leske DA, Burke JP, Hodge DO. Birth prevalence of visually significant infantile cataract in a defined U.S. population. Ophthalmic Epidemiol 2003; 10(2): 67-74.

23 Li LH, Li N, Zhao JY, Fei P, Zhang GM, Mao JB et al. Findings of perinatal ocular examination performed on 3573, healthy full-term newborns. Br J Ophthalmol 2013; 97(5): 588-591.
24 Limburg H, Gilbert C, Hon DN, Dung NC, Hoang TH. Prevalence and causes of blindness in children in Vietnam. Ophthalmology 2012; 119(2): 355-361.

25 Lu Q, Zheng Y, Sun B, Cui T, Congdon N, Hu A et al. A population-based study of visual impairment among pre-school children in Beijing: the Beijing study of visual impairment in children. Am J Ophthalmol 2009; 147(6): 1075-1081.

26 Luteijn JM, Dolk H, Addor MC, Arriola L, Barisic I, Bianchi F et al. Seasonality of congenital anomalies in Europe. Birth Defects Res A Clin Mol Teratol 2014; 100(4): 260-269.

27 Nirmalan PK, Vijayalakshmi P, Sheeladevi S, Kothari MB, Sundaresan K, Rahmathullah L. The Kariapatti pediatric eye evaluation project: baseline ophthalmic data of children aged 15 years or younger in Southern India. Am J Ophthalmol 2003; 136(4): 703-709.

28 Pi L-H, Chen L, Liu Q, Ke N, Fang J, Zhang S et al. Prevalence of eye diseases and causes of visual impairment in school-aged children in Western China. J Epidemiol 2012; 22(1): 37-44.

29 Rahi JS, Botting B. British Congenital Cataract Interest G. Ascertainment of children with congenital cataract through the National Congenital Anomaly System in England and Wales. Br J Ophthalmol 2001; 85(9): 1049-1051.

30 SanGiovanni JP, Chew EY, Reed GF, Remaley NA, Bateman JB, Sugimoto TA et al. Infantile cataract in the collaborative perinatal project: prevalence and risk factors. Arch Ophthalmol 2002; 120(11): 1559-1565.

31 Shirima S, Lewallen S, Kabona G, Habiyakare C, Massae P, Courtright P. Estimating numbers of blind children for planning services: findings in Kilimanjaro, Tanzania. Br J Ophthalmol 2009; 93(12): 1560-1562.

32 Stewart-Brown SL, Haslum MN. Partial sight and blindness in children of the 1970 birth cohort at 10 years of age. J Epidemiol Community Health 1988; 42: 17-23.

33 Stoll C, Alembik Y, Dott B, Roth MP. Congenital eye malformations in 212,479 consecutive births. Ann Genet 1997; 40(2): 122-128.

34 Xiao B, Fan J, Deng Y, Ding Y, Muhit M, Kuper H. Using key informant method to assess the prevalence and causes of childhood blindness in Xiu'shui County, Jiangxi Province, Southeast China. Ophthalmic Epidemiol 2011; 18(1): 30-35.

35 Abrahamsson M, Magnusson G, Sjostrom A, Popovic Z, Sjostrand J. The occurrence of congenital cataract in western Sweden. Acta Ophthalmol Scand 1999; 77(5): 578-580.

36 Haargaard B, Wohlfahrt J, Fledelius HC, Rosenberg T, Melbye M. Incidence and cumulative risk of childhood cataract in a cohort of 2.6 million Danish children. Invest Ophthalmol Vis Sci 2004; 45(5): 1316-1320.

37 Rahi JS, Dezateux C. British Congenital Cataract Interest Group Measuring and interpreting the incidence of congenital ocular anomalies: lessons from a national study of congenital cataract in the UK. Invest Ophthalmol Vis Sci 2001; 42 (7):1444-1448.

38 Wirth MG, Russell-Eggitt IM, Craig JE, Elder JE, Mackey DA. Aetiology of congenital and paediatric cataract in an Australian population. Br J Ophthalmol 2002; 86(7): 782-786.

39 Forster JE, Abadi RV, Muldoon M, Lloyd IC. Grading infantile cataracts. Ophthalmic Physiol Opt 2006; 26(4): 372-379.

40 Anstice NS, Thompson B. The measurement of visual acuity in children: an evidence-based update. Clin Exp Optom 2014; 97(1): 3-11. 
41 Sommer A, Davidson FR. Assessment and control of vitamin A deficiency: the Annecy Accords. J Nutr 2002; 132(9 Suppl): 2845s-2850s.

42 Birch EE, Stager D, Leffler J, Weakley D. Early treatment of congenital unilateral cataract minimizes unequal competition. Invest Ophthalmol Vis Sci 1998; 39(9): 1560-1566.

43 Lambert SR, Lynn MJ, Reeves R, Plager DA, Buckley EG, Wilson ME. Is there a latent period for the surgical treatment of children with dense bilateral congenital cataracts? J AAPOS 2006; 10(1): 30-36.
44 Medsinge A, Nischal KK. Pediatric cataract: challenges and future directions. Clin Ophthalmol 2015; 9: 77-90.

45 Sengpiel F. Plasticity of the visual cortex and treatment of amblyopia. Curr Biol 2014; 24(18): R936-R940.

46 Pizzarello L, Abiose A, Ffytche T, Duerksen R, Thulasiraj R, Taylor H et al. VISION 2020: The Right to Sight: a global initiative to eliminate avoidable blindness. Arch Ophthalmol 2004; 122(4): 615-620.

47 Thylefors B. A global initiative for the elimination of avoidable blindness. Community Eye Health 1998; 11: 1-3.

Supplementary Information accompanies this paper on Eye website (http://www.nature.com/eye) 